\title{
Preprocessing Images Algorithm without Gaussian Shaped Particles for PIV Analysis and Imaging Vortices on the Epicardial Surface
}

\author{
Daria Mangileva ${ }^{1,2}$, Alexander Kursanov ${ }^{2}$, Alena Tsvetkova ${ }^{3}$, Olesya Bernikova ${ }^{3}$, Alexey \\ Ovechkin ${ }^{3}$, Maria Grubbe ${ }^{3}$, Jan Azarov ${ }^{3}$ and Leonid Katsnelson ${ }^{2}$ \\ ${ }^{1}$ Ural Federal University, Mira Street 19, Ekaterinburg, 620002, Russia \\ ${ }^{2}$ Institute of Immunology and Physiology, Pervomayskaya Street 106, Ekaterinburg, 620049, Russia \\ ${ }^{3}$ Department of Cardiac Physiology, Institute of Physiology, Komi Science Center, Pervomayskaya Street 50, \\ Syktyvkar, 167000, Russia
}

\begin{abstract}
Image preprocessing for vector field building with Particle Image Velocimetry can significantly affect the quality of the resulting information. It is especially relevant when it comes to tasks where there are no auxiliary markers in the video recordings. Such tasks include the study of the mechanical behavior of the epicardial surface on video recordings obtained on an open heart. In particular, this study aims to visualize the vortex-like mechanical movements that can occur on the heart during fibrillation. The knowledge gained about the deformation of the epicardial wall can help in better understanding the pathological processes. However, due to the intense movement of the heart and the presence of blood on surface, the application of the necessary small markers is rather difficult, and the use of luminous chemicals would harm physiological functioning. Moreover, these videos contain motion artifacts that complicate further analysis with Particle Image Velocimetry. In this paper, an image preprocessing algorithm was proposed. It is based on approximate tracking individual fragments using the Mean Squared Error for the matrix. The result is binary images where small points are built instead of each fragment. In this study, the proposed algorithm showed better results in comparison with the most suitable filtering methods for specific frames, namely, the Sobel filter and the Canny edge detector. This can be partially explained by the higher density of vector fields due to the absence of unreliable vectors. Thus, the proposed method, unlike others, allows to get vector fields with visible vortex-like mechanical movements.

Keywords

PIV, preprocessing, vortex, heart surface, filtering
\end{abstract}

\section{Introduction}

Ventricular fibrillation (VF) often complicates myocardial infarction and results in sudden cardiac death. VF prediction and prevention constitutes an important research challenge. Despite important insights into arrhythmogenesis have been provided (Varró et al., 2021) [1], this problem remains largely unsolved thus far, which warrants further studies of VF mechanisms. One of probably underestimated arrhythmogenic mechanisms might be mechanical interaction between ischemic and normal tissues that can cause multiple proarrhythmic changes via a so-called mechanoelectrical feedback. A method of assessment of mechanical interaction between adjacent myocardial regions in vivo is needed for the evaluation of this phenomenon. Such a method should be free of significant interventions that can affect the functional myocardial properties.

GraphiCon 2021: 31st International Conference on Computer Graphics and Vision, September 27-30, 2021, Nizhny Novgorod, Russia EMAIL: alpendwarf@gmail.com (D. Mangileva); a.kursanov@iip.uran.ru (A. Kursanov); as.tsvetkova@mail.ru (A. Tsvetkova); bernikovaog@gmail.com (O. Bernikova); alexlena@ inbox.ru (A. Ovechkin); grubbe.me@gmail.com (M. Grubbe); j.azarov@gmail.com (J. Azarov); 1.katsnelson@iip.uran.ru (L. Katsnelson ).

ORCID: 0000-0002-4879-7130 (D. Mangileva); 0000-0002-0563-0808 (A. Kursanov); 0000-0003-4268-153X (A. Tsvetkova); 0000-00029780-1503 (O. Bernikova); 0000-0003-1821-9492 (A. Ovechkin); 0000-0002-6032-2461 (M. Grubbe); 0000-0001-9553-9110 (L. Katsnelson) GitHub: https://github.com/DariaMangileva/Pre-processing-Images-Algorithm-Without-Gaussian-Shaped-Particles-for-PIV-Analysis (c) (i) 2021 Copyright for this paper by its authors.

Use permitted under Creative Commons License Attribution 4.0 International (CC BY 4.0).

CEUR Workshop Proceedings (CEUR-WS.org) 
Particle Image Velocimetry (PIV) [2] has become one of the most popular methods for various fields of research that apply the analysis of the motion of various objects in video recordings. Since the beginning of its active use and until now, this approach has been actively refined and today it has software implementations, for example, in the MATLAB environment [3] and the OpenPIV Python library [4]. In particular, this method can be used for assessing myocardial deformation [5]. The main point of this method is that it measures particle flows by comparing two consecutive frames. If one briefly describes this method, then at the beginning there is a cross-correlation between the given interrogation windows. Interrogation window sizes and cross-correlation methods can vary. For the most accurate motion estimation, the direct cross-correlation (DCC) algorithm is usually used [6]. Figure 1 shows a simplified diagram of this algorithm.

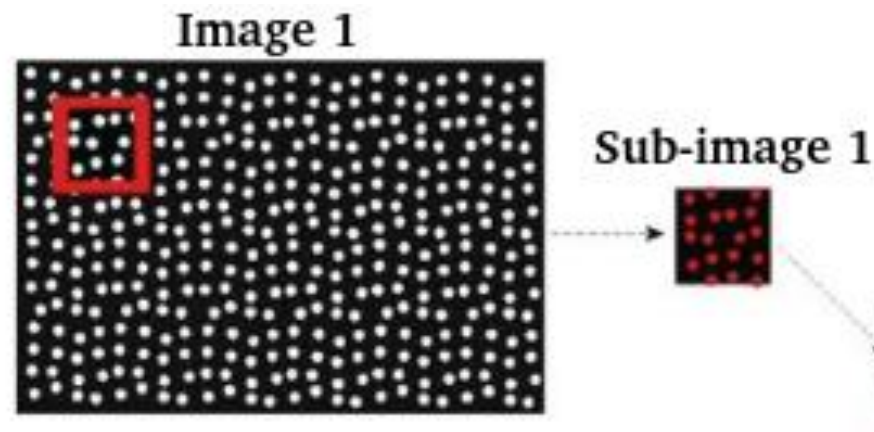

Image 2

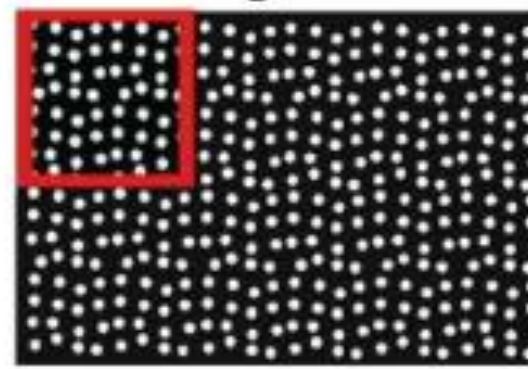

\section{Sub-image 2}

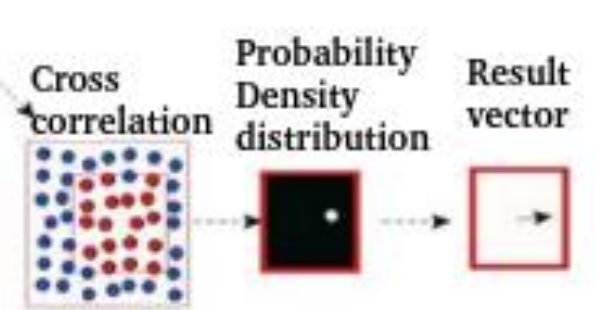

Figure 1: Simplified scheme of the direct cross-correlation algorithm

The main idea is that the interrogation window is set on the first frame (sub-image 1), and then on the second frame. An area of the sub-image 1 size, which has the maximum correlation with sub-image 1 , is searched for on the first frame within the large interrogation window (sub-image 2). The twodimensional Gaussian regression method has proven itself well for determining the maximum in the cross-correlation matrix [7]. However, the study by Nobach and Honkanen [7] proved that this method worked best for images with Gaussian-shaped particles. Therefore, in preparation for the PIV analysis, various methods are used to ensure that the frames have an appropriate look. So, for example, a special laser sheet is used to study the motion of particles in the fluid, which highlights them and facilitates the preprocessing of frames [3]. Recently, however, PIV has become increasingly used to study the motion of various surfaces. It should be noted that for this kind of research, expensive cameras with a high frame capture rate are usually used to minimize motion artifacts, but they are not always available due to limited financial resources [8]. Also, in many cases, auxiliary markers are used. They take on the role of particles and contribute to the best detection of peaks in the cross-correlation matrix, but this is not always possible $[9,10]$.

The current study aims to obtain information using PIV analysis on the mechanical movement on the ventricular epicardium of the pig heart in the presence of ischemia and VF. It is assumed that electrical vortices, which cause VF, lead to viral mechanical movements, which may be reflected on the epicardial surface. 
In this paper, preprocessing algorithm was proposed, partially inspired by the DCC algorithm, which can improve the quality of the information obtained from the video recordings of the experiment, without resorting to the application of small markers, the use of glowing chemicals and expensive cameras.

\section{Materials and methods}

All programming operations performed in this study were applied using the Python programming language. For reproducibility of the experiment results, programs and video files were uploaded to the GitHub.

\subsection{Experiment description}

The study conformed to the Guide for the Care and Use of Laboratory Animals, 8th Edition published by the National Academies Press (USA) 2011, the guidelines from Directive 2010/63/EU of the European Parliament on the protection of animals used for scientific purposes and was approved by the ethical committee of the Institute of Physiology of the Komi Science Centre, Ural Branch of Russian Academy of Sciences.

The experiment was performed in a male pig ( $36.5 \mathrm{~kg}$ body weight, 2 months old). The animal was anesthetized with zoletil (ZOLETIL ${ }^{\circledR} 100$, Virbac S.A., Carros, France,10-15 mg/kg, i.m.), xylazine (Interchemie, Castenray, Netherlands, $0.5 \mathrm{mg} / \mathrm{kg}$, i.m.) and propofol (Norbrook Laboratories Ltd., Newry, Northern Ireland, UK, $1 \mathrm{mg} / \mathrm{kg}$, i.v.), intubated and mechanically ventilated. The heart was accessed via a midsternal incision. Myocardial ischemia was induced by occlusion of the left anterior descending coronary artery just distal to the first diagonal branch origin. Limb lead ECGs were continuously recorded throughout the experiment in order to control ECG parameters and arrhythmias development. Small angular markers were placed on the epicardium of the ventricles for analysis of the ventricular surface movements. Markers were distributed over the epicardial surface as uniformly as possible, covering both normally perfused and ischemic areas. The video registration of anterior wall movement of the heart was done before coronary occlusion and during ischemia until VF developed in the first minute, after which the animal was euthanized under deep anesthesia by an intravenous potassium chloride injection.

A video recording of the pig's open heart was made on a single-lens reflex camera with a relatively low frame capture rate (50 frames per second). Considering that the contraction of the heart is quite intense, there are motion artifacts in these videos. Moreover, to obtain more detailed information on the mechanical behaviour of the epicardial surface, it is necessary to use smaller interrogation windows for the DCC algorithm. Therefore, in this case, it is necessary to apply more small markers located quite close to each other, but this is troublesome due to the constant presence of blood on the surface of the heart. Thus, this task requires a more thorough preprocessing of frames.

\subsection{Preprocessing algorithm}

Since it has already been proven that two-dimensional Gaussian regression works best on images with clearly defined Gaussian-shaped particles [7], the main purpose of this preprocessing is to bring frames to such a form. The first frame of the video of the experiment was divided into a grid in the region of the heart, which was determined manually. Given the movement of the heart during contractions, the area of interest was slightly less than the surface area of the epicardium. The cell size was $16 \times 16$ pixels. Figure 2 shows an image of a heart with a superimposed virtual mesh. 


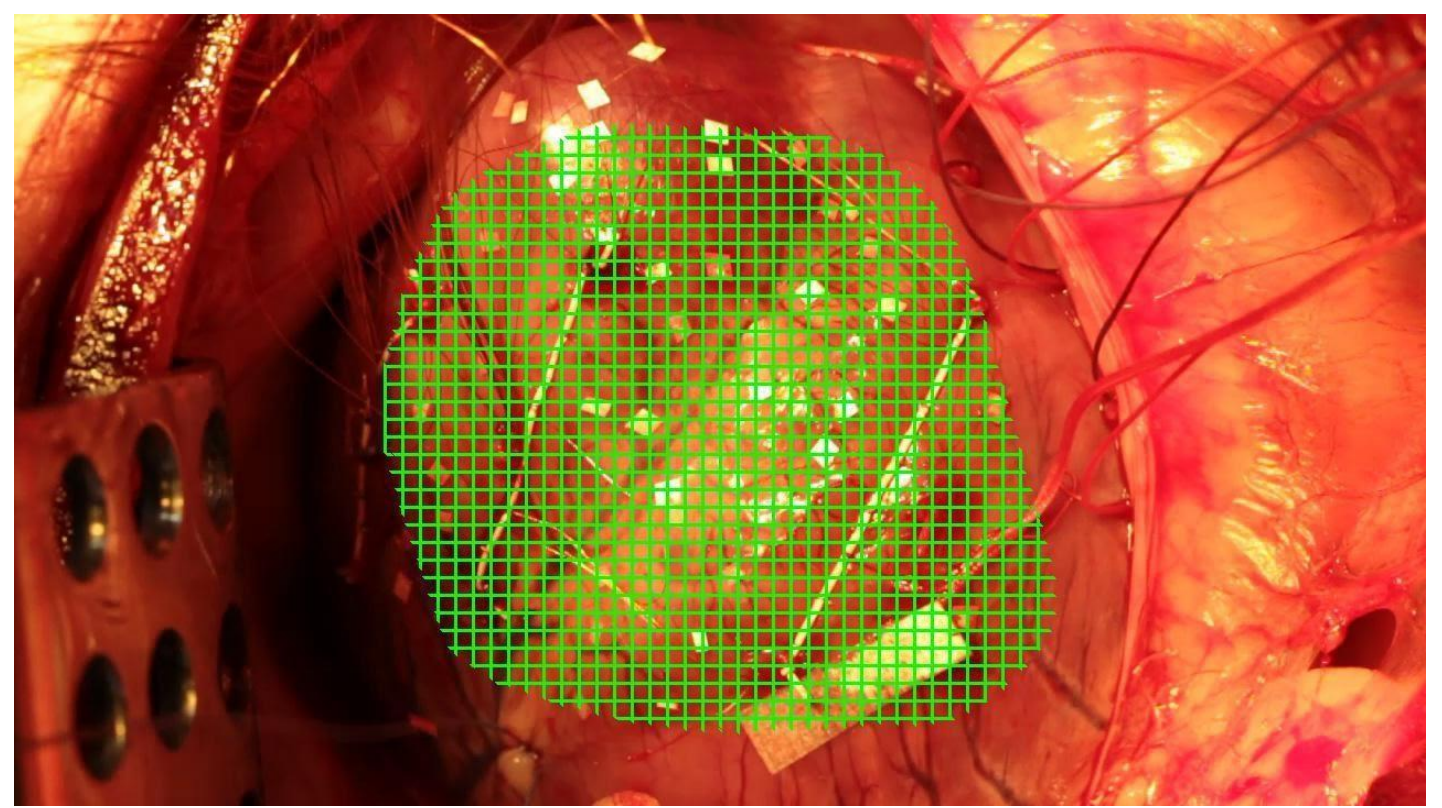

Figure 2: Image of the virtual mesh superimposed on the epicardial surface

A point was drawn in the center of each cell. Next, each of these cells was monitored using a simple proposed algorithm. The essence of this approach is that on the next frame cell " $\mathrm{B}$ " is built (Figure 3) located in the same place as cell " $\mathrm{A}$ ", but the size of cell " $\mathrm{B}$ " is $32 \times 32$. Next, a $16 \times 16$ square " $\mathrm{A}$ " " is built around each point of cell "B" and it is compared with cell "A" using the Mean Squared Error (MSE) for the matrix [11] (1)

$$
\text { MSE }=\frac{1}{\mathrm{k}} \sum_{\mathrm{i}=0}^{\mathrm{n}} \sum_{\mathrm{j}=0}^{\mathrm{m}}\left\|\mathrm{A}_{\mathrm{i}, \mathrm{j}}-\mathrm{A}_{\mathrm{i}, \mathrm{j}}^{\prime}\right\|^{2}
$$

It is worth noting that since the input images were in the RGB format, the final MSE was calculated as an average across all channels.

The coordinates of the center of the cell corresponding to the smallest value of the standard deviation were recorded. Then the algorithm was repeated according to the same principle, but concerning the newly selected point. The algorithm scheme is shown in Figure 3.

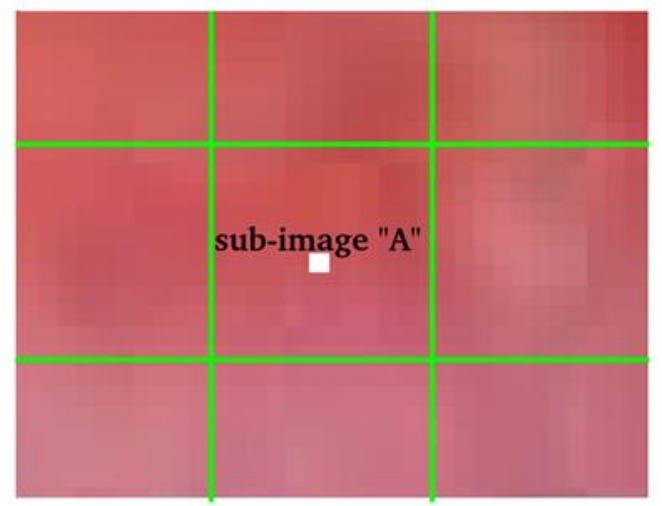

frame 1

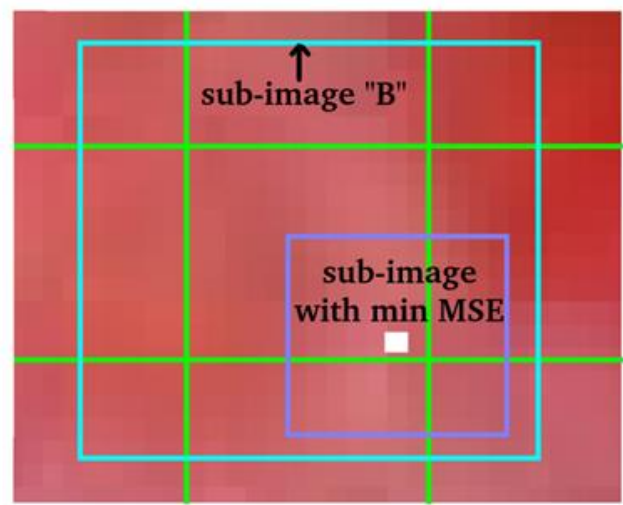

frame 2

Figure 3: Scheme of the proposed algorithm

An example of the image obtained after the above procedure is shown in Figure 4. 


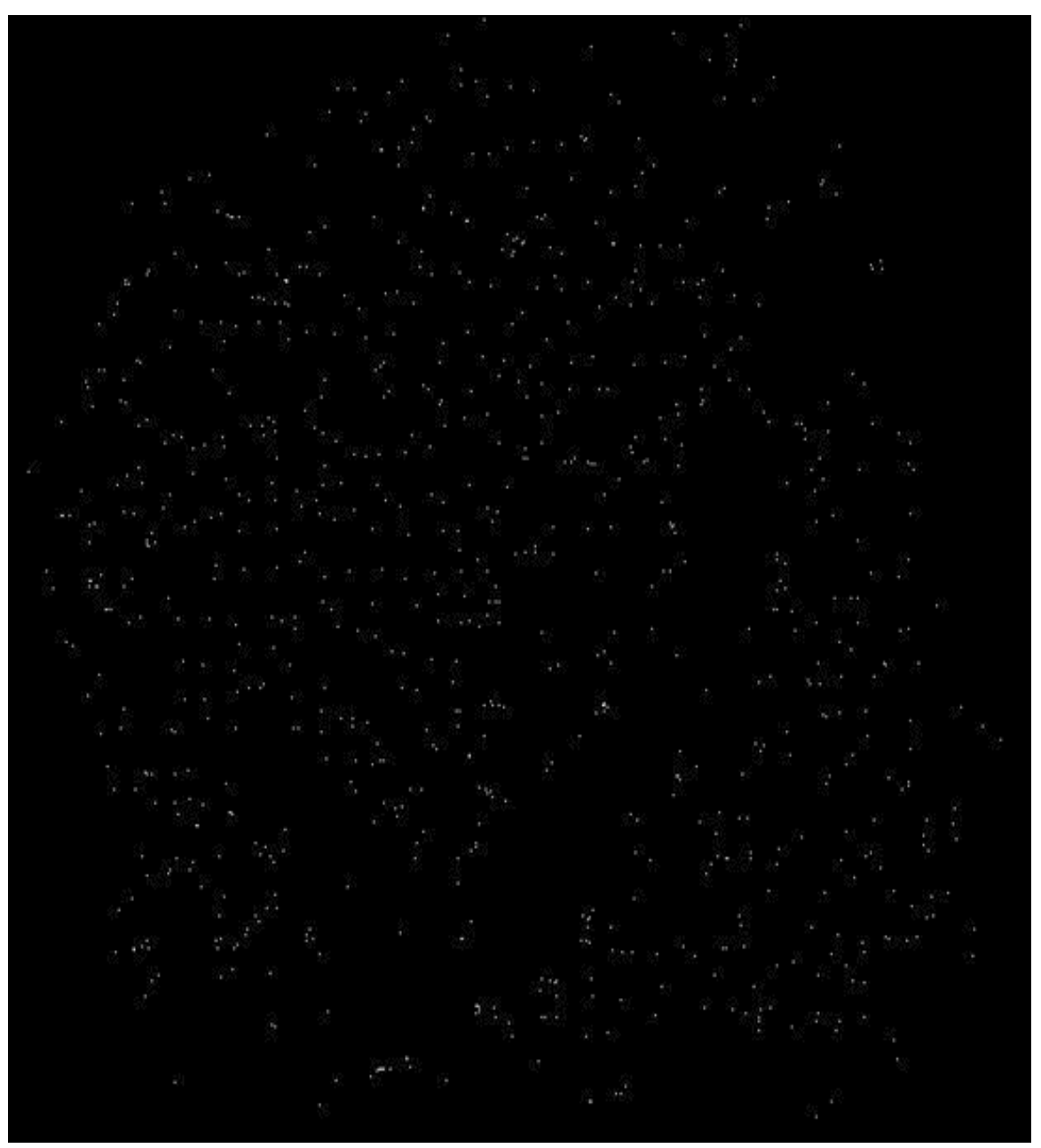

Figure 4: Sample image after suggested processing

\subsection{Selection of filtering methods for comparison with the proposed algorithm}

Since the preprocessing method described above is computationally expensive, it must be justified by comparing it with the most suitable classical filtering for these frames. Figure 5 shows the image of the same frame after different filtering. For this study, various filters were selected, such as a threshold filter [12], median filter [13], Gaussian filter [14], Canny edge detection [15], and Sobel filter [16]. It should be noted that the filter parameters, and in particular the value of the subsequent threshold filtering and Gaussian (sigma) for Canny edge detection, were chosen optimally for a specific task.

It is obvious from Figure 5 that Canny edge detection and the Sobel filter are best suited for this task, since it is necessary to visualize the phenomena occurring on the entire surface of the epicardium, and the threshold filter, median filter, and Gaussian filter lead to a significant loss of information. Since only information about the epicardium remains on the image in the method described in the previous paragraph, for the purity of the experiment, a mask was applied to all filtered frames in such a way that the entire image area except for the heart became black. 

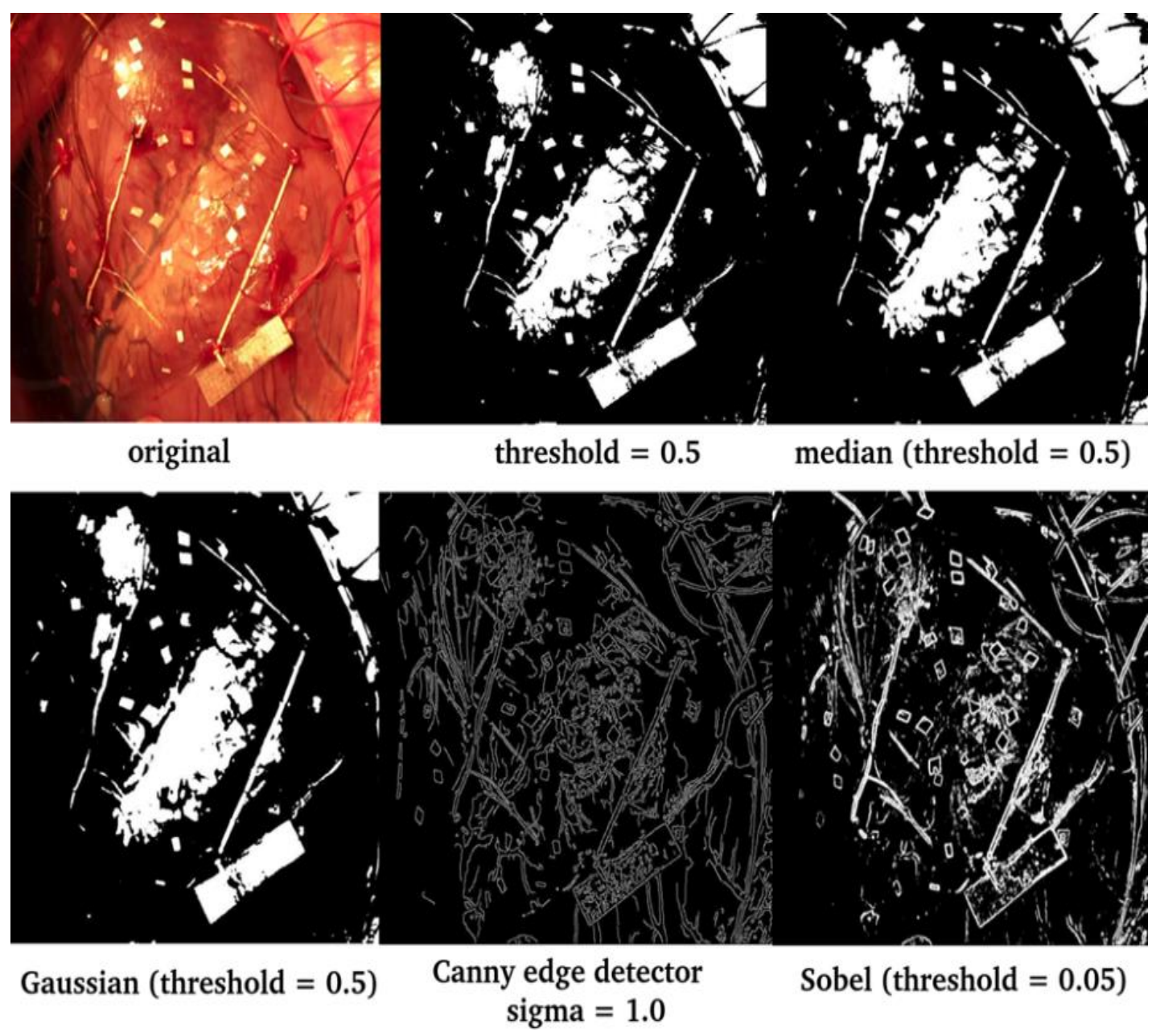

Figure 5: Examples of frames obtained after various filtering

\subsection{PIV analysis}

On the processed images, a vector field was constructed using the PIV method implemented in the OpenPIV Python library[4]. For the DCC cross-correlation algorithm, a small window of 16x16 and a large window of $32 \times 32$ were chosen. A two-dimensional Gaussian regression method was chosen for estimating the subpixel location of the peak. If there were less than 1.05 ratios of the first peak to the second largest peak, then these vectors were determined as unreliable and were reset to zero.

\subsection{Estimating a vector field with the Swirling Strength Criterion}

To compare several preprocessing methods over the entire period of the experiment, the maximum Swirling Strength Criterion (SSC) developed by Zhou et al. (1999) [17] and having a software implementation in the Vortex Fitting software package [18] was calculated. It defines a vortex core to be the region where $\overline{\mathrm{D}}$ has complex eigenvalues. It is based on the idea that the velocity gradient tensor in Cartesian coordinates can be decomposed as follows (2):

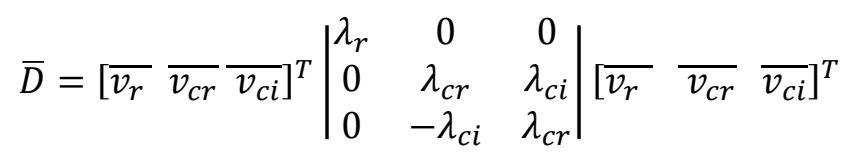

where $\lambda_{r}$ is the real eigenvalue related to the eigenvector $\overline{v_{r}}$, 
$\lambda_{c r} \pm i \lambda_{c i}$ is the complex conjugate pair of complex eigenvalues related to the eigenvectors $\overline{v_{r}} \pm i \overline{v_{c i}}$

The strength of this swirling motion can be quantified by $\lambda_{c i}$, which is called the local swirling.

The SSC value is capable, in a sense, of numerically assessing the severity of the vortex, since it was originally invented for their detection. This criterion also affects the size of the current vortex [17]. However, it should be noted that the presence of any twists in the vector field, particularly the mechanical phenomena that arise during the natural contraction of the heart, should also have some value of the SSC [17]. However, in the presence of fibrillation, more pronounced swirling should appear on the vector field, which in theory should lead to an increase in SSC [17].

To identify a more global trend in SSC changes, the graphs were smoothed using a moving average [19] equal to 8 seconds (400 frames).

\section{Results}

Figure 6 shows a smoothed SSC versus time dependency graph for different preprocessing methods. A compressed electrocardiogram is shown below the graph.

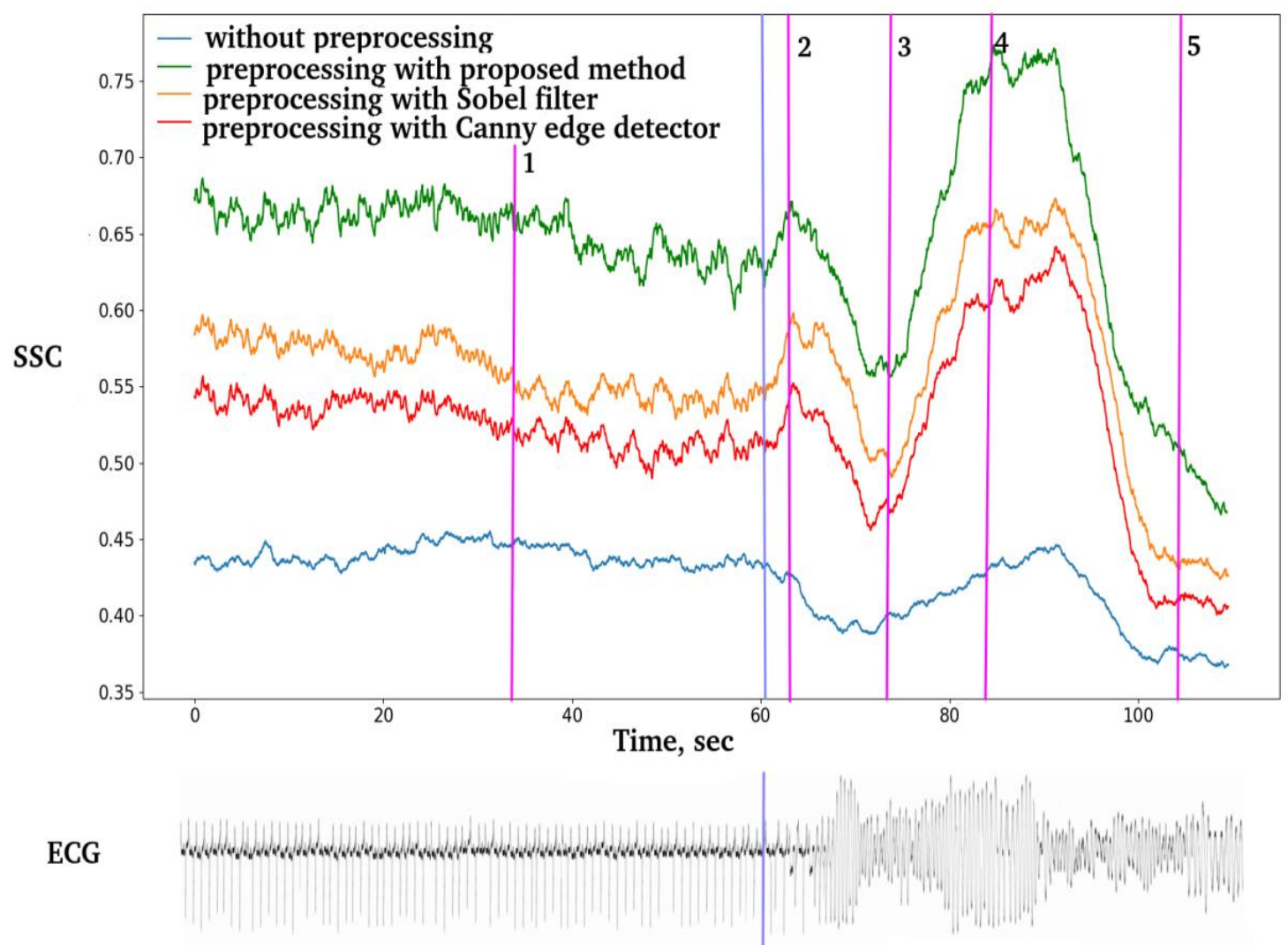

Figure 6: Change in the swirling strength criterion over time. The pink lines mark arbitrary points that are located in sufficiently different zones of the graph. The violet line on the graphs and ECG marks the moment of occlusion onset with smoothing being considered.

Figure 7 shows examples of images of vector fields for the marked points in Figure 6. It should be noted that the selection of frames was made with the smoothing of the graph. 


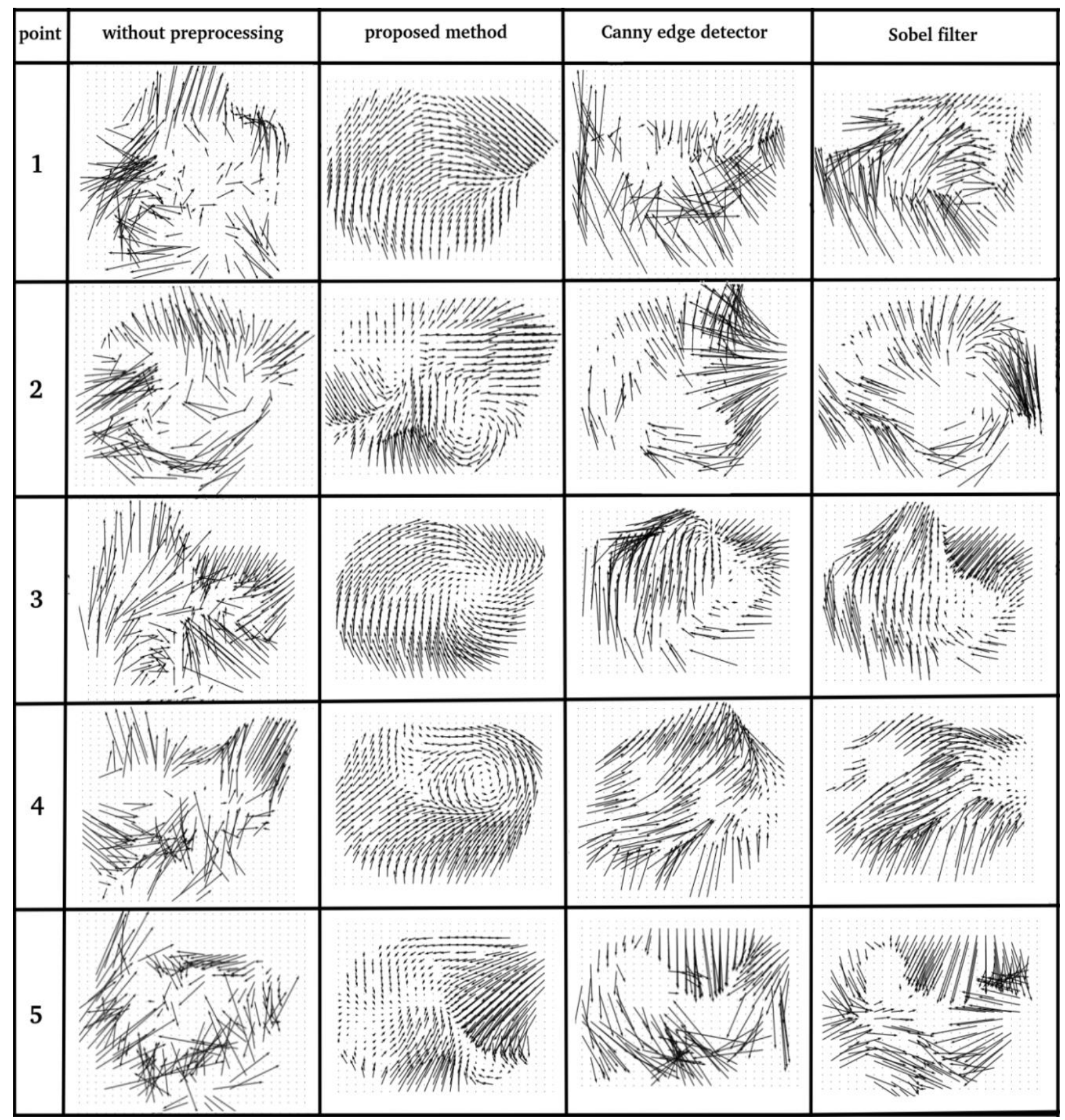

Figure 7: Image of vector fields for different points and preprocessing methods

Table 1 shows the percentage of unreliable vectors for each method.

Table 1

Percentage of unreliable vectors

\begin{tabular}{cccc}
\hline $\begin{array}{c}\text { Without } \\
\text { preprocessing }\end{array}$ & $\begin{array}{c}\text { Proposed } \\
\text { method }\end{array}$ & $\begin{array}{c}\text { Canny edge } \\
\text { detector }\end{array}$ & $\begin{array}{c}\text { Sobel } \\
\text { filter }\end{array}$ \\
\hline $25 \%$ & $0 \%$ & $21 \%$ & $13 \%$ \\
\hline
\end{tabular}

Table 2 shows the differences between the maximum and minimum values of the graphs in Figure 6 after occlusion. 
Table 2

Difference between the maximum and minimum SSC values at the post-occlusion gap for different preprocessing methods

\begin{tabular}{cccc}
\hline $\begin{array}{c}\text { Without } \\
\text { preprocessing }\end{array}$ & $\begin{array}{c}\text { Proposed } \\
\text { method }\end{array}$ & $\begin{array}{c}\text { Canny edge } \\
\text { detector }\end{array}$ & $\begin{array}{c}\text { Sobel } \\
\text { filter }\end{array}$ \\
\hline 0.08 & 0.31 & 0.23 & 0.25 \\
\hline
\end{tabular}

\section{Conclusions}

The method proposed in this article improves the quality of the analysis of vector fields obtained using PIV. This can be seen from the graph in Figure 6. The curve obtained using this method is located higher than others. Thus, the probability of vortex detection [17] increases due to the higher SSC value. Moreover, it has the highest sensitivity judging by the values in Table 2 . This can be explained by the fact that the vector fields built on these preprocessed images have a higher density due to the absence of unreliable vectors (Table 1). From the graph in Figure 6, Table 1, and Table 2, one can see that the density of the vector field directly affects the level of the SSC value and the sensitivity of the graph after occlusion. Thus, the best results are obtained with the proposed method, the next is the Sobel filter, then comes the Canny edge detector and raw video. On this basis, this study confirms the fact that the two-dimensional Gaussian regression method works best on black and white images with particles. Though this method can give errors, even with the subsequent use of DCC analysis and two-dimensional Gaussian regression, one can see in Figure 7 that the vector fields built on images preprocessed using this method look quite harmonious compared to other vector fields. Moreover, it is possible to visually discern vortices on them, especially at points 2 and 4 . The vortices at points 3 and 5 on the vector fields are less noticeable. This can be explained by the limitations of the proposed method by the size of the virtual grid (Figure 2). Thus, one can conclude that this method can be used for solutions of such problems without additional aids.

\section{Acknowledgements}

The study was supported by Grant No. 21-14-00226 of the Russian Science Foundation Supercomputer URAN of IMM UrB RAS was used for calculations.

\section{References}

[1] Varró, András, et al. "Cardiac transmembrane ion channels and action potentials: cellular physiology and arrhythmogenic behavior." Physiological Reviews 101.3 (2021): 1083-1176. doi: https://doi.org/10.1152/physrev.00024.2019

[2] R.J. Adrian, Particle-imaging techniques for experimental fluid mechanics, Annual Review of Fluid Mechanics 23 (1991): 261-304. doi: http://dx.doi.org/ 10.1146/annurev.fl.23.010191.001401

[3] W. Thielicke, E.J. Stamhuis, PIVlab - towards user-friendly, affordable and accurate digital particle image velocimetry in MATLAB, Journal of Open Research Software 2 (2014): e30. doi: http://dx.doi.org/10.5334/jors.bl

[4] OpenPIV Group, OpenPIV: a python package for PIV image analysis, 2014. URL: https://openpiv.readthedocs.io/en/latest/

[5] Jacob, Athira, Ganapathy Krishnamurthi, and Manikandan Mathur. "Estimation of myocardial deformation using correlation image velocimetry." BMC medical imaging 17.1 (2017): 1-13. doi: https://doi.org/10.1186/s12880-017-0195-7.

[6] H. Huang, D. Dabiri, M. Gharib, On errors of digital particle image velocimetry, Measurement Science and Technology 8 (1997): 1427-1440. doi: http:// dx.doi.org/10.1088/0957-0233/8/12/007

[7] H. Nobach, M. Honkanen, Two-dimensional Gaussian regression for sub-pixel displacement estimation in particle image velocimetry or particle position estimation in particle tracking 
velocimetry, Experiments in Fluids 38 (2005): 511-515. doi: http://dx.doi.org/10.1007/s00348005-0942-3

[8] A. Nemes, T. Dasari, J. Hong, M. Guala, F. Coletti, Snowflakes in the atmospheric surface layer: observation of particle-turbulence dynamics, Journal of Fluid Mechanics 814 (2017): 592. doi: https://doi.org/10.1017/jfm.2017.13

[9] K. Kuok, P.C. Chiu, Application of particle image velocimetry (PIV) for measuring water velocity in laboratory sedimentation tank, IRA International Journal of Technology \& Engineering 9(3): 16-26 (2017). doi: http://dx.doi.org/10.21013/jte.v9.n3.p1

[10] C.J. Legleiter, P.J. Kinzel, Surface flow velocities from space: Particle image velocimetry of satellite video of a large, sediment-laden river, Frontiers in Water 3 (2021). doi: https://doi.org/10.3389/frwa.2021.652213

[11] A. Mood, F. Graybill, D. Boes, Introduction to the Theory of Statistics, 3rd ed., McGraw-Hill, 1950, p. 229.

[12] L. Shapiro, G.C. Stockman, Computer Vision, Prentice Hall, 2002.

[13] R. Boyle, R. Thomas, Computer Vision: A First Course, Blackwell Scientific Publications, 1988, pp. 32-34.

[14] H.J. Blinchikoff, A.I. Zverev, Filtering in the Time and Frequency Domains, SciTech Publishing, (2001). doi: https://doi.org/10.3986/ac.v48i2.7269

[15] J. Canny, A computational approach to edge detection, IEEE Transactions on Pattern Analysis and Machine Intelligence 8(6) (1986): 679-698. doi: 10.1109/TPAMI.1986.4767851

[16] R. Duda, P. Hart, Pattern classification and scene analysis, John Wiley and Sons, 1973, pp. 271272. doi: $10.2307 / 2286028$

[17] J. Zhou, R.J. Adrian, S. Balachandar, T.M. Kendall, Mechanisms for generating coherent packets of hairpin vortices in channel flow, Journal of Fluid Mechanics 387 (1999): 353-96. doi: http://dx.doi.org/10.1017/s002211209900467x

[18] G. Lindner, Y. Devaux, S. Miskovic, VortexFitting: A post-processing fluid mechanics tool for vortex identification, SoftwareX 12 (2020): 100604. doi: 10.1016/j.softx.2020.100604

[19] Y.L. Chou, Statistical Analysis, Holt International, 1975, section 17.9. 